\title{
Planet accretion and the abundances of lithium isotopes
}

\author{
J. Montalbán ${ }^{1,2}$ and R. Rebolo ${ }^{1,3}$ \\ 1 Instituto de Astrofísica de Canarias, 38200 La Laguna (Tenerife), Spain \\ e-mail: (jmontalb, rrl)@ll.iac.es \\ 2 Osservatorio Astronomico di Roma, 00040 Monteporzio, Italy \\ e-mail: montalbn@coma.mporzio.astro.it \\ 3 Consejo Superior de Investigaciones Científicas, Spain
}

Received 17 December 2001 / Accepted 5 March 2002

\begin{abstract}
Planet accretion onto solar type stars may significantly change the stellar atmospheric abundances of ${ }^{6} \mathrm{Li}$ and ${ }^{7} \mathrm{Li}$ if it takes place after the star has arrived at the main sequence. Ingestion of planets at an earlier phase will not affect theses abundances because of extensive pre-main sequence mixing and burning. We present quantitative estimates of the main sequence evolution of stellar surface lithium abundances after planet ingestion. At solar metallicities, for stellar masses in the range $\sim 1.3-1.1 M_{\odot}$, a large fraction of the ingested ${ }^{6} \mathrm{Li}$ is likely to be preserved during the whole main sequence lifetime according to the standard model. Preservation of the less fragile ${ }^{7} \mathrm{Li}$ isotope occurs in a larger mass range $\sim 1.3-0.9 M_{\odot}$. At high metallicities typical of planet host stars, the ranges of masses are slightly reduced essentially due to the thicker convective zones. Further reduction is expected if non-standard processes cause extra-mixing of material below the base of the convective zone, but even in this case there are stellar masses where both isotopes are significantly preserved. We conclude that there is a range of stellar effective temperature where migration and accretion of planets and planetary material can be empirically tested using high-resolution spectroscopy of the lithium isotopes.
\end{abstract}

Key words. stars: evolution - stars: abundances

\section{Introduction}

The accretion of planets or planetary material onto stars have been considered as a possible explanation of the high metallicity frequently found in planet host stars (Laughlin \& Adams 1997; Gonzalez 1997, 1998, 2001; Sandquist et al. 1998; Laughlin 2000; Santos et al. 2000; Murray et al. 2001; Pinsonneault et al. 2001). The very recent detection of the ${ }^{6} \mathrm{Li}$ isotope in the atmosphere of the solartype star HD 82943 (Israelian et al. 2001), which hosts a planetary system (Mayor et al. 2001), has also been interpreted as evidence for accretion of planets. Jupiter-like planets are expected to preserve their original content of the lithium isotopes, while stars like HD 82943 with a mass $\sim 1.1 M_{\odot}$ destroy, via nuclear reactions with protons, all its ${ }^{6} \mathrm{Li}$ (and a small fraction of the more robust ${ }^{7} \mathrm{Li}$ ) during the pre-main sequence (PMS) evolution (see e.g. Proffitt \& Michaud 1989). It has been argued that ingestion and dilution of planetary material in HD 82943 after its superficial convective zone receded to the mainsequence location allowed long-term preservation of the newly acquired abundances of lithium isotopes. In fact, according to standard models for this type of stars, the

Send offprint requests to: J. Montalbán, e-mail: montalbn@coma.mporzio.astro.it base of the convective zone does not reach the temperatures needed for ${ }^{6} \mathrm{Li}$ and ${ }^{7} \mathrm{Li}$ burning $\left(T \geq 2.2 \times 10^{6} \mathrm{~K}\right.$ and $T \geq 2.6 \times 10^{6} \mathrm{~K}$, respectively). The rate of destruction of ${ }^{6} \mathrm{Li}$ by protons has a temperature dependence similar to that of ${ }^{7} \mathrm{Li}$, but on much shorter time scale (i.e. 100 times shorter at $1 M_{\odot}$ in the standard model).

In this paper, we examine in quantitative terms the possibility that the dilution of one or several Jupiter-like planets in the convective envelope of a main sequence star could appreciably change the observed abundance of ${ }^{6} \mathrm{Li}$ and ${ }^{7} \mathrm{Li}$. In the framework of the standard model we predict the evolution of these isotopic abundances in stars with masses ranging from 1.3 to $0.6 M_{\odot}$. We also discuss the effect due to some transport processes which cause extra-mixing below the convective zone (see for review Michaud \& Charbonneau 1991). We will show that there is a narrow range of mass (or $T_{\text {eff }}$ ) where the accretion of planetary material could produce an observable increase of ${ }^{6,7} \mathrm{Li}$ abundance during a significant fraction of the lifetime of the star.

\section{Models}

The stellar models have been computed with the updated version of the ATON2.0 code (Ventura et al. 1998). 
We constructed models with masses ranging from 0.6 to $1.3 M_{\odot}$ with a step of $\Delta M=0.05 M_{\odot}$, and followed the evolution from the pre-main sequence to 7 Gyr. These models use a mixing length theory (MLT) description of convective transport with a parameter of mixing length $\alpha=1.6$, as given by the solar calibration (Ventura et al. 1998; Montesinos et al. 2001). Two different chemical compositions were adopted: a) initial solar helium abundance of $Y_{\odot}=0.28$ and heavy-elements mass fraction of $Z_{\odot}=0.02([\mathrm{Fe} / \mathrm{H}]=0.0)$ (Montesinos et al. 2001), b) $Z=0.04$, with $\Delta Y / \Delta Z=2$, that is, $[\mathrm{Fe} / \mathrm{H}]=+0.34$.

The code used in our model computations allows to include gravitational settling of helium and thermal diffusion, but it does not consider the diffusion of metals. Diffusion generally produces a reduction of the convection zone, however, Turcotte et al. (1998) showed that the convective envelopes in models with metal diffusion are deeper than in models including only helium settling. Furthermore, their results indicate (see their Fig. 4) that for the higher masses considered in this paper $(M \leq$ $\left.1.3 M_{\odot}\right)$ the mass of the convective envelope of a nondiffusion model is actually closer to the full-diffusion one than to a model incorporating He settling only (in fact, the non-diffusion and full-diffusion curves overlap). Because of that, we decided to use stellar structures obtained without including diffusion. Notice also that, a proper treatment of diffusion (Turcotte et al. 1998) has no significant impact on the evolution of lithium abundance in stars with $M \leq 1.3 M_{\odot}$. The nuclear burning rates of ${ }^{6} \mathrm{Li}$ and ${ }^{7} \mathrm{Li}$ in the star's convective envelope were computed for each temporal step in evolution using NACRE nuclear reaction tables (Angulo et al. 1999).

Masses for the engulfed sub-stellar companions ranged, from $1 M_{\mathrm{J}}{ }^{1}$ to $40 M_{\mathrm{J}}$. This upper mass limit was adopted to ensure the complete preservation of ${ }^{6} \mathrm{Li}$ (Pozio 1991; Nelson et al. 1993), but, in practice, the maximum mass of the accreted planet was constrained to be less than the mass in the convective zone of the star under consideration.

The hypothesis and approximations adopted in our study are: $i$ ) planet accretion takes place just after the star arrives on the main sequence. The analysis presented here does not change if the planet is engulfed at any other moment during the main sequence lifetime. Such case can be dealt by taking into account that the temperature, mass of the convective envelope and the stellar surface lithium abundance will be different since they decrease as age increases. ii) The planet is completely dissolved in the stellar convective envelope. As shown by numerical simulations (Sandquist et al. 1998), the reliability of this assumption depends on the mass of the planet $\left(M_{\mathrm{Pl}}\right)$ and on the mass of the convective envelope $M_{\mathrm{CZ}}$, and also strongly depends on the details of the internal structure of the planet. The smaller the $M_{\mathrm{CZ}}$, the lower the fraction of planet dissolved in the convective zone. That means that, at least for the higher planet masses under consideration, the quantity of

\footnotetext{
${ }^{1}$ Jupiter mass, $M_{\mathrm{J}}=9.55 \times 10^{-4} M_{\odot}$.
}

lithium provided by the planet must be reduced in a factor equal to the fraction of the planet consumed in the star's convective envelope. iii) The engulfment of a planet has no significant effect in the structure of the star. iv) The planet is instantly mixed in the convective zone of the star. Given that the diffusion coefficient in the convective zone is of the order of $10^{13} \mathrm{~cm}^{2} \mathrm{~s}^{-1}$, the mixing time is lower than the temporal step in the evolution computation.

The stellar models were followed from the PMS, but we will not use the surface abundance of ${ }^{6} \mathrm{Li}$ and ${ }^{7} \mathrm{Li}$ given by the models because it is well known that they overestimate PMS lithium destruction (D'Antona \& Mazzitelli 1997). For masses lower than $1.4 M_{\odot}$ it is expected that no ${ }^{6} \mathrm{Li}$ exists after the PMS (Proffitt \& Michaud 1989). As regards surface ${ }^{7} \mathrm{Li}$ abundance we adopted the values observed in stars of the Pleiades Cluster (Soderblom et al. 1993). So the stellar surface abundances of lithium isotopes at the moment of the engulfment of the planet are: $N\left({ }^{6} \mathrm{Li}\right) / N(H)=0$ and $N\left({ }^{7} \mathrm{Li}\right) / N(H)=F\left(T_{\text {eff }}\right)_{\text {Pleiades. }}$. These abundances in the planets are assumed equal to the meteoritic values. That is: $N\left({ }^{7} \mathrm{Li}\right)_{0} / N(H)=2 \times 10^{-9}$, and $N\left({ }^{7} \mathrm{Li}\right)_{0} / N\left({ }^{6} \mathrm{Li}\right)_{0}=12$.

Following Burrows \& Sharp (1999) we assume an Anders \& Grevesse (1989) solar chemical composition for both the planet and the star. In this case, the difference between the abundance of the isotope $J$ in the star's convective zone before $(\mathrm{s})$ and after $(\mathrm{s}+\mathrm{p})$ the dilution of the planet can be written as:

$\epsilon(J)_{\mathrm{s}+\mathrm{p}}-\epsilon(J)_{\mathrm{s}}=\log \left(\frac{x}{x+1} \cdot(1-f)\right)-\log f$.

Where $\epsilon(J)=\log (N(J) / N(H))+12, x=M_{\mathrm{Pl}} / M_{\mathrm{CZ}}$, and $f$ is the depletion factor of $J$ in the convective zone just before the absorption of the planet. So the change in the stellar surface abundance depends strongly on the factor $f$, and it is evident from Eq. (1) that the lithium abundance enhancement will be significant only if the mass fraction of the isotope $J$ in the planet is much higher than in the stellar convective envelope. If the engulfed planet has lost a significant part of its initial content of hydrogen, the mass fraction of lithium in the planet is larger than in the solar chemical composition case. Therefore, also the enhancement of atmospheric lithium abundance will be larger, and the results presented here would be a lower limit.

\section{Evolution of Li provided by planetary material}

\subsection{Standard model framework}

The standard model assumes that no exchange of material occurs between the convective envelope and the stable radiative interior of the star. Therefore, the surface chemical composition reflects only the composition of the well mixed convective envelope. In this subsection we present the evolution of lithium in the stellar surface due to the nuclear burning taking place in the convective envelope. 


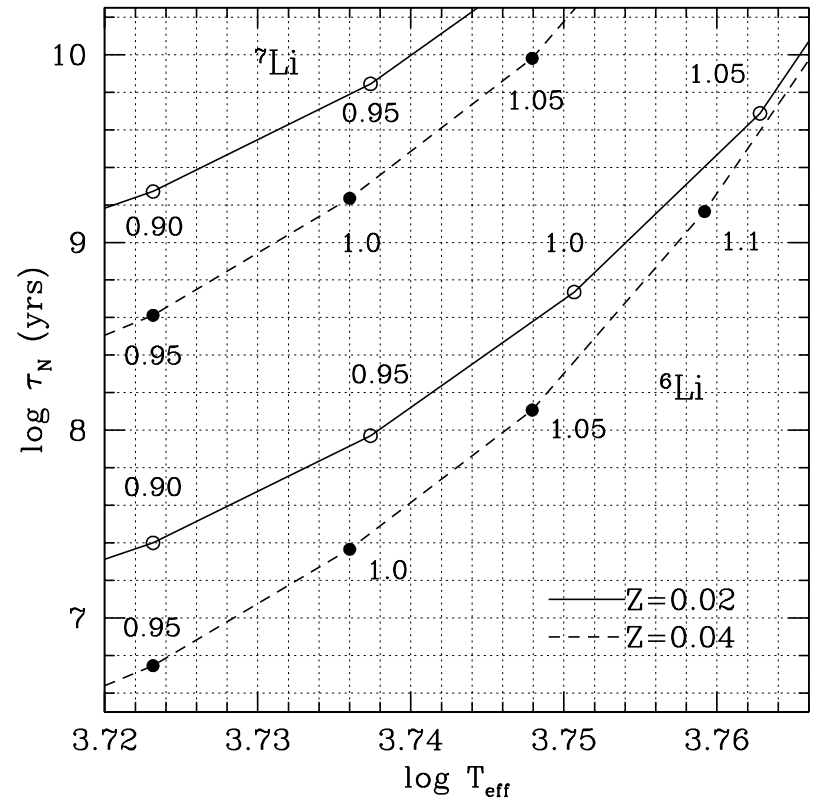

Fig. 1. ${ }^{6} \mathrm{Li}$ (lower curves) and ${ }^{7} \mathrm{Li}$ (upper curves) lifetime against $(p, \alpha)$ nuclear reactions $\left(N(\mathrm{Li}) / N(\mathrm{Li})_{0}=\exp \left(-t / \tau_{\mathrm{N}}\right)\right)$ as a function of effective temperature. The curves correspond to standard models at $10^{8}$ yr with two different metallicities: $[\mathrm{Fe} / \mathrm{H}]=0.0$ (solid lines) and $[\mathrm{Fe} / \mathrm{H}]=+0.34$ (dashed lines). The numbers close to the symbols (empty circles for solar metallicity and full circles for $[\mathrm{Fe} / \mathrm{H}]=+0.34)$ indicate the mass of the stellar model.

Assuming solar chemical composition, a planet of $1 M_{\mathrm{J}}$ supplies $\sim 1.4 \times 10^{44}$ atoms of ${ }^{6} \mathrm{Li}$ and $\sim 1.7 \times 10^{45}$ atoms of ${ }^{7} \mathrm{Li}$. What is the evolution of these atoms during the main sequence lifetime of the star? The nuclear time scale, $\tau_{\mathrm{N}}$, is an estimation of how long these atoms could survive to the nuclear burning in the convective envelope. $\tau_{\mathrm{N}}$, is defined as the time needed to decrease the superficial abundance of the the isotope $J$ by a factor $e$, and it has been obtained from the nuclear reaction rates at each layer in the convective zone, and assuming instantaneous mixing. In Fig. 1 we plot $\tau_{\mathrm{N}}$ for ${ }^{6} \mathrm{Li}$ and ${ }^{7} \mathrm{Li}$ at $100 \mathrm{Myr}$ as a function of effective temperature. We plot the curves corresponding to two different values of metallicity. We see that for $\log T_{\text {eff }} \geq 3.76(\sim 5700 \mathrm{~K})$, a large fraction of the ${ }^{6} \mathrm{Li}$ from the planet will survive for a long time in the convective envelope of the star. The value of $\tau_{\mathrm{N}}$ given in the plot is a minimum, since during the main sequence lifetime, the temperature at the bottom of the convective zone decreases, and so the nuclear time scale increases. The effect of increasing the metallicity is to reduce the nuclear time scale at a given $T_{\text {eff }}$. A factor 2 higher metallicity implies a factor 3 lower time scale at $5500 \mathrm{~K}$, a factor 2 at $5700 \mathrm{~K}$ and only a reduction of $10 \%$ for $T_{\text {eff }}$ higher than $5800 \mathrm{~K}$.

Figure 2 presents the evolution (based on models and hypothesis described in Sect. 2) of ${ }^{6} \mathrm{Li}$ and ${ }^{7} \mathrm{Li}$ abundance for several stellar masses, and for two values of metallicity: $[\mathrm{Fe} / \mathrm{H}]=0.0$ (left panel) and $[\mathrm{Fe} / \mathrm{H}]=+0.34$ (right panel). That temporal dependence is obtained computing the nuclear depletion of ${ }^{6} \mathrm{Li}$ and ${ }^{7} \mathrm{Li}$ in the stellar convective envelope for each temporal step in the evolution and assuming instantaneous mixing. We plot only values of lithium abundances corresponding to times longer than the time of planet accretion, and lower than the dredge-up of the convective envelope. In the upper panels, we plot the curves corresponding to the ${ }^{6} \mathrm{Li}$ that result from the accretion of a planet with $\sim 1,5$, and $10 M_{\mathrm{J}}$ in stars with masses between 0.9 and $1.2 M_{\odot}$ (for 1.25 and $1.3 M_{\odot}$ the enhancement of ${ }^{6} \mathrm{Li}$ is slightly larger because the mass of the convective envelope is smaller, and the curves are parallel to those of $1.2 M_{\odot}$ ). This preliminary and simple modelling provides strong support for the interpretation by Israelian et al. (2001) of the ${ }^{6} \mathrm{Li}$ detection in HD 82943. According to our models, assuming a $T_{\text {eff }} \sim 6000 \mathrm{~K}$, age of $5 \mathrm{Gyr}$ and metallicity $[\mathrm{Fe} / \mathrm{H}] \sim 0.32$ (Santos et al. 2000) this star has a mass of $\sim 1.2 M_{\odot}$. As can be seen in Fig. 2 right panel, it should preserve any ingested ${ }^{6} \mathrm{Li}$ during all the main sequence lifetime.

The ${ }^{6} \mathrm{Li}$ abundances plotted in Fig. 2 were obtained assuming a complete dissolution of the planet in the stellar convective zone. For solar metallicity, the masses of the convective envelope in stars with 1.2, 1.1 and $1.0 M_{\odot}$ are $0.006 M_{\odot}, 0.017 M_{\odot}$ and $0.034 M_{\odot}$, respectively. Numerical simulations by Sandquist et al. (1998) indicate that as the mass of the convective envelope decreases, the fraction of planet that is dissolved is reduced. If only 0.3 of a Jupiter-like planet is dissolved in the convective zone of a $1.2 M_{\odot}$ star, the corresponding curve of ${ }^{6} \mathrm{Li}$ will only decrease in $\sim 0.2$ dex.

Figure 2 shows that there is a mass range in which ${ }^{6} \mathrm{Li}$ could survive. In fact, for stars with masses larger than $1.1 M_{\odot}\left(\right.$ for $Z_{\odot}$, or $1.15 M_{\odot}$ for $\left.Z=2 Z_{\odot}\right)$ the superficial abundance of ${ }^{6} \mathrm{Li}$ does not change with age. The reason is that during their main sequence lifetime, the temperature at the base of the convective zone is not high enough to produce efficient burning of ${ }^{6} \mathrm{Li}$. As the stellar mass decreases, the depth of the convective zone increases, and then, even if a large quantity of planetary material is dissolved in the stellar envelope, ${ }^{6} \mathrm{Li}$ will be depleted in a short time scale $\left(\sim 10^{8} \mathrm{yr}\right)$.

The lower panels of Fig. 2 presents the predictions for ${ }^{7} \mathrm{Li}$ according to standard model. Note that, opposite to ${ }^{6} \mathrm{Li}$, the ${ }^{7} \mathrm{Li}$ isotope is depleted on the PMS only by a small factor, and that stars with masses larger than $0.9 M_{\odot}$ will not deplete a significant amount during the main sequence lifetime. If these stars were able to dissolve $10 M_{\mathrm{J}}$ in their convective envelope, the effect on the abundance would be to introduce only a small enhancement, 0.2 dex at most. The reason is shown in Eq. (1), where we see that the increase of abundance depends strongly on the depletion factor of ${ }^{7} \mathrm{Li}$ in the convective envelope before the accretion of the planetary material.

\subsection{Impact of non-standard transport processes}

Observations of lithium in open clusters show that the atmospheric abundance decreases during the main sequence 


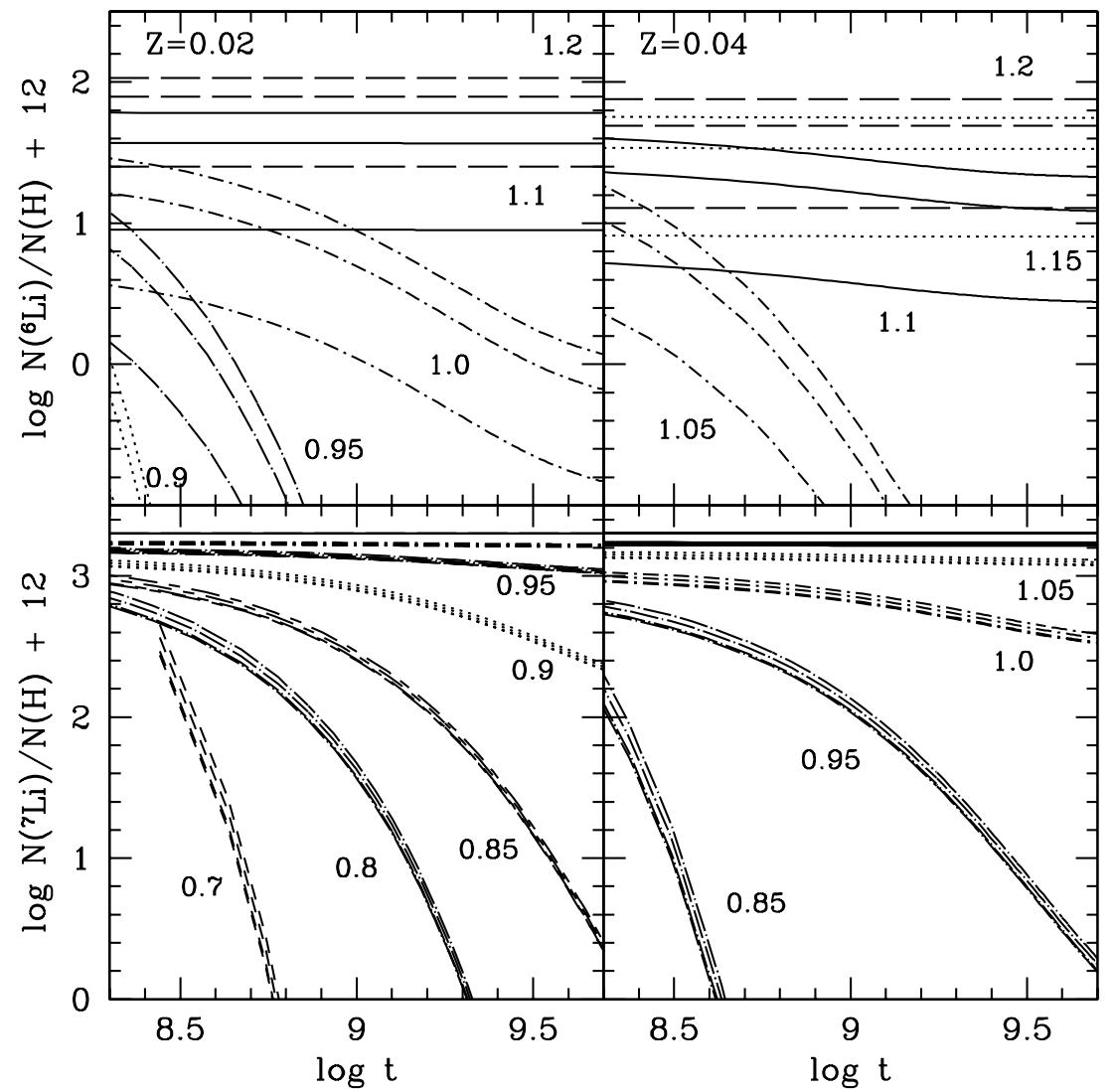

Fig. 2. Evolution of ${ }^{6} \mathrm{Li}$ (upper panels) and ${ }^{7} \mathrm{Li}$ (lower panels) provided by dissolution of planets in the convective envelope of stars with masses from 1.2 to $0.7 M_{\odot}$ (numbers indicate the mass of the star) and metallicities: $[\mathrm{Fe} / \mathrm{H}]=0.0$ (left panels) and $[\mathrm{Fe} / \mathrm{H}]=+0.34$ (right panels). ${ }^{6} \mathrm{Li}$ : for each stellar model there are three curves corresponding to (from down to top) $\sim 1,5$ and $10 M_{\mathrm{J}}$ of planetary material dissolved. ${ }^{7} \mathrm{Li}$ : as for ${ }^{6} \mathrm{Li}$, but we also plot the curve corresponding to no planet accretion. The ${ }^{7} \mathrm{Li}$ curves corresponding to: 1.0, 1.1 and $1.2 M_{\odot}$ (left panel), and 1.1, 1.15 and $1.2 M_{\odot}($ right panel) are concentrated in the top of the respective figures.

lifetime. In order to explain this main-sequence depletion different mixing processes are invoked (e.g. Michaud \& Charbonneau 1991), and we should consider their effect on the evolution of ${ }^{6} \mathrm{Li}$ in the stellar mass range where standard models predict complete preservation. Among these processes are: a) microscopic diffusion, that consists roughly in the sink of heavy elements with respect to the light ones due to the gravitational potential; b) turbulent diffusion which causes mixing of material between the convective zone and the deep layers where the temperature is high enough to burn lithium.

Concerning the first one, the diffusion velocities are similar for ${ }^{6} \mathrm{Li}$ and ${ }^{7} \mathrm{Li}$, but effects of microscopic diffusion are negligible for the effective temperature and ages considered here (e.g. Richer \& Michaud 1993). Turcotte et al. (1998) showed that for $1.3 M_{\odot}$ star, the effect of microscopic diffusion on $\mathrm{Li}$ is at most a depletion by a factor 0.55 , and only after 2 Gyr. In order to test the effect of turbulent mixing we have considered a diffusion coefficient produced by internal waves that fits the evolution of ${ }^{7} \mathrm{Li}$ abundances between the Pleiades and M 67 clusters quite well (Montalbán \& Schatzman 2000): ${ }^{6} \mathrm{Li}$ would decrease only in 0.2 dex after 5 Gyr in a $1.2 M_{\odot}$ $\operatorname{star}\left(Z_{\odot}\right)$. However, in a $1.1 M_{\odot}\left(Z_{\odot}\right)$ star, ${ }^{6} \mathrm{Li}$ will decrease 0.6 dex in the first Gyr and 1.7 dex after 5 Gyr. Of course, these results depend strongly on the behaviour of the diffusion coefficient close to the base of the convective zone. We recall that the diffusion coefficient produced by internal waves has large amplitude at the CZ boundary, and then it decreases rapidly downwards. Therefore, the results are very sensitive to the distance between the base of the convective zone and the ${ }^{6} \mathrm{Li}$ burning layer. We have also tested a parametric turbulent diffusion coefficient $D_{\mathrm{T}}=D_{0}\left(\rho_{\mathrm{cz}} / \rho\right)^{3}$ as proposed by Proffitt \& Michaud (1991) ( $\rho_{\mathrm{cz}}$ is the density at the base of convective zone). A value of $D_{0} \sim 1.5 \times 10^{3} \mathrm{~cm}^{2} \mathrm{~s}^{-1}$ produces, after 5 Gyr, a ${ }^{7} \mathrm{Li}$ depletion of: 1.6 dex for $1 M_{\odot}$ $(\sim 5800 \mathrm{~K}), 0.2 \mathrm{dex}$ for $1.1 M_{\odot}(\sim 6000 \mathrm{~K})$, and $<0.1 \mathrm{dex}$ for $1.2 M_{\odot}(\sim 6100 \mathrm{~K})$ (these values are in good agreement with estimations by Pinsonneault et al. 2001 using mixing induced by rotation), and causes a ${ }^{6} \mathrm{Li}$ depletion of 0.9 dex at $T_{\text {eff }} \sim 6000 \mathrm{~K}, 0.05$ dex at $T_{\text {eff }} \sim 6100 \mathrm{~K}$, and a complete depletion after $1 \mathrm{Gyr}$ for $T_{\text {eff }} \sim 5800 \mathrm{~K}$.

From observations it is inferred that the efficiency of non-standard mixing decreases with the age in the main-sequence. So what happens if the accretion of the planetary material takes place in a ${ }^{7} \mathrm{Li}$ depleted star at a sufficently old age for extra-mixing to be inefficient? 
The stellar ${ }^{7} \mathrm{Li}$ abundance could be significanly enhanced for a long period of time. For instance, if a $1 M_{\mathrm{J}}$ planet were dissolved in our Sun's convective envelope would produce an enhancement of $0.3 \mathrm{dex}$, if $10 M_{\mathrm{J}}$ were accreted the corresponding increase would be 0.9 dex.

\section{Conclusions}

The results presented here show that the accretion of planetary material by a solar-type star will produce an enhancement of the stellar surface abundance of lithium which could be observable during a large fraction of the main sequence lifetime. Within the framework of the standard model, the $T_{\text {eff }}$ range where the ingested ${ }^{6} \mathrm{Li}$ could be detected is $5900-6400 \mathrm{~K}$. The upper limit is imposed by observational evidence given by the Li-Gap in the Hyades (Boesgaard \& Tripicco 1986). The lower limit is the result of direct convective mixing and should increase about 100 or $200 \mathrm{~K}$ when extra-mixing processes are considered. The $T_{\text {eff }}$ range where ${ }^{7} \mathrm{Li}$ would survive is larger. Therefore, a very valuable test for planetary migration/accretion scenarios can be provided by high-resolution spectroscopic observations of ${ }^{6,7} \mathrm{Li}$ in planet host stars.

\section{References}

Anders, E., \& Grevesse, N. 1989, Geochim. Cosmochim. Acta, 53, 197

Angulo, C., et al. 1999, Nucl. Phys. A., 656, 3

Boesgaard, A. M., \& Tripicco, M. J. 1986, ApJ, 302, L49
Burrows, A., \& Sharp, C. M. 1999, ApJ, 512, 843

D'Antona, F., \& Mazzitelli, I. 1997, Mem. S. A. It., 68, 807

Gonzalez, G. 1997, MNRAS, 285, 403

Gonzalez, G. 1998, A\&A, 334, 221

Gonzalez, G., Laws, C., Tyagi, S., \& Reddy, B. E. 2001, AJ, 121,432

Israelian, G., Santos, N. C., Mayor, M., \& Rebolo, R. 2001, Nature, 411, 163

Laughlin, G. 2000, ApJ, 545, 1064

Laughlin, G., \& Adams, F. C. 1997, ApJ, 491, L51

Mayor, M., Naef, D., Pepe, F., et al. 2001, ESO Press Release, $07 / 01$

Michaud, G., \& Charbonneau, P. 1991, Space Sci. Rev., 57, 1

Montalbán, J., \& Schatzman, E. 2000, A\&A, 354, 943

Montesinos, B., Thomas, J. H., Ventura, P., \& Mazzitelli, I. 2001, MNRAS, 326, 877

Nelson, L. A., Rappaport, S., \& Chiang, E. 1993, ApJ, 413, 364

Pinsonneault, M. H., DePoy, D. L., \& Coffee, M. 2001, ApJ, 556, L59

Proffitt, C. R., \& Michaud, G. 1991, ApJ, 380, 238

Proffitt, C. R., \& Michaud, G. 1989, ApJ, 346, 976

Pozio, F. 1991, Mem. S. A. It, 61

Richer, J., \& Michaud, G. 1993, ApJ, 416, 312

Sandquist, E., Taam, R. E., Lin, D. N. C., \& Burkert, A. 1998, ApJ, 506, L65

Santos, N. C., Israelian, G., \& Mayor, M. 2000, A\&A, 363, 228

Soderblom, D. R., Jones, B. J., Balachandran, S., et al. 1993, AJ, 106, 1059

Turcotte, S., Richer, J., \& Michaud, G. 1998, ApJ, 504, 559

Ventura, P., Zeppieri, A., Mazzitelli, I., \& D'Antona, F. 1998, A\&A, 334, 953 